\title{
Parameter Conditions for Global Stability of FAST TCP
}

\author{
Kyungmo Koo, Joon-Young Choi, and Jin S. Lee
}

\begin{abstract}
In this letter, we study the global asymptotic stability of FAST TCP in the presence of network feedback delays. Based on a continuous-time dynamic model of FAST TCP and a static approximation of queuing delay at the link, we derive stability conditions for FAST TCP. The derived conditions are explicitly appeared as tuning parameter conditions of FAST TCP, and hence can be satisfied in a distributed way. The simulation results illustrate the validity of the conditions for the global asymptotic stability.
\end{abstract}

Index Terms-Congestion control, FAST TCP, stability.

\section{INTRODUCTION}

$\mathbf{F}$ AST TCP is a new TCP congestion control algorithm for high-speed long-latency networks and uses the queuing delays as congestion measure [1]. When the queuing delays are used for congestion measure, the users at the sources are able to avoid packet losses to detect congestion, hence the algorithm with this mechanism increases utilization and is scalable in the large bandwidth-delay product network [1]. To fully utilize these advantages of FAST TCP, it is all the more important to ensure that the network with FAST TCP is stable and always stays near the equilibrium state.

Extensive experimental and several theoretical efforts have been devoted to the study on the stability property of FAST TCP in [1], [2], [3], [4], [5], [6]. Local stability of FAST TCP is studied for the case of a single link without network feedback delays in [1], and for the homogeneous network with network feedback delays in [2]. Recently, with a new accurate link model, local stability of FAST TCP is analyzed for a single-link network in [3]. In [4], the local stability condition is derived in terms of not only the parameter $\gamma$ but also $\alpha$. To ensure that the algorithm converges asymptotically to its equilibrium from any initial state, the global stability analysis is a significant open challenge but there are few results in this area. Global stability of FAST TCP is studied for the case of a single link network without network feedback delays in [2] and with network feedback delays in [5].

In this letter, we study the global asymptotic stability of FAST TCP in a single-link single-source network with network feedback delays. In [5], the stability condition is derived in terms of the tuning parameter $\alpha$. As a complementary work of [5], we consider the parameter $\gamma$ as well as $\alpha$ for the global stability of FAST TCP. The derived conditions agree with the local stability results of [3], [4] and can be satisfied in

Manuscript received October 5, 2007. The associate editor coordinating the review of this letter and approving it for publication was I. Venieris.

K. Koo and J. S. Lee are with the Department of Electronic and Electrical Engineering, Pohang University of Science and Technology, Korea (e-mail: \{pumpkins, jsoo\}@ postech.ac.kr).

J.-Y. Choi is with the Department of Electronic Engineering, Pusan National University, Korea (e-mail: jyc@pusan.ac.kr).

Digital Object Identifier 10.1109/LCOMM.2008.071633. a decentralized way because each source can independently tune its own parameters based on its link status.

\section{NETWORK MODEL}

To avoid mathematical difficulty and complexity, we focus on the case of a single-link single-source network in which a pair of sender and receiver node is connected through a single bottleneck link. The link has a finite transmission capacity $c$ and is assumed to have infinite buffering storage. Associated with the link is the queuing delay $p(t)$ and with the source is the congestion window $w(t)$. We assume at time $t$ that the source observes as a feedback signal the measured queuing delay $q(t) \triangleq p\left(t-\tau^{b}\right)$, where $\tau^{b}$ denotes the backward delay in the feedback path from link to source, and the link observes incoming TCP packets $w\left(t-\tau^{f}\right)$, where $\tau^{f}$ denotes the forward delay from source to link. The round trip time (RTT) $T(t)$ is defined for the source as $T(t) \triangleq d+q(t)$, where $d$ is the constant round trip propagation time.

FAST TCP periodically updates its congestion window as follows [1]

$$
w(t+\Delta)=\gamma\left(\frac{\text { baseRTT }}{\operatorname{RTT}} w(t)+\alpha\right)+(1-\gamma) w(t),
$$

where baseRTT is the minimum RTT observed, $\alpha$ is the tuning parameter that determines the total number of backlogs in routers, $\gamma \in(0,1]$ is the tuning parameter that determines the step size of the algorithm, and $\Delta$ is the window update period. Using the Euler's method $\dot{w}(t) \approx \frac{w(t+\Delta)-w(t)}{\Delta}$, and defining $\gamma_{e} \triangleq \frac{\gamma}{\Delta}$, FAST TCP can be expressed as a differential equation

$$
\dot{w}(t)=\gamma_{e}\left(-w(t)+\frac{d}{d+q(t)} w(t)+\alpha\right) .
$$

Based on the self-clocking property of TCP, a static approximation model for the link is given as [2], [5]

$$
\frac{w\left(t-\tau^{f}\right)}{d+p(t)}\left\{\begin{array}{ll}
=c & \text { if } p(t)>0 \\
\leqslant c & \text { if } p(t)=0
\end{array} .\right.
$$

Assuming the network is under congestion, i.e. $p(t)>0$, the link model can be expressed at the source side as

$$
\frac{w(t-T(t))}{d+q(t)}=c
$$

from $q(t)=p\left(t-\tau^{b}(t)\right)$ and the round trip feedback delay $\tau^{f}+\tau^{b}(t)=T(t)$. Note that this static link model adequately describes the RTT period of delay between the congestion window $w(\cdot)$ and measured queuing delay $q(\cdot)$, where $q(t)$ is the delayed feedback information of the congestion caused by $w(t-T(t))$.

The closed-loop system under congestion consists of the congestion window adjustment rule of each source described 
by (1) and the queuing delay described by (2). We formulate the closed-loop system as

$$
\begin{aligned}
\dot{w}(t) & =\gamma_{e}\left(-w(t)+\frac{d}{d+q(t)} w(t)+\alpha\right) \\
& =\gamma_{e}\left(-\frac{q(t)}{d+q(t)} w(t)-c d+\alpha+c d\right) \\
& =\gamma_{e}\left(-\frac{q(t)}{d+q(t)} w(t)-\frac{d}{d+q(t)} w(t-T)+\alpha+c d\right),
\end{aligned}
$$

where the last equality is from the link model (2). The corresponding equilibrium point $w^{*}$ is uniquely determined as $w^{*}=\alpha+c d$, and consequently $q^{*}=\frac{\alpha}{c}$ from (2). Making a change of variable in terms of the window size error $\tilde{w}(t) \triangleq w(t)-w^{*}=w(t)-\alpha-c d$, we can express the closed-loop system of FAST TCP network as follows:

$$
\dot{\tilde{w}}(t)=\gamma_{e}\left(-\frac{q(t)}{d+q(t)} \tilde{w}(t)-\frac{d}{d+q(t)} \tilde{w}(t-T)\right) .
$$

\section{BOUNDEDNESS}

As a preliminary to the subsequent analysis, we state that $w(t)$ and $q(t)$ are bounded with respect to $t$. The following lemma shows that $w(t)$ and $q(t)$ are uniformly bounded above.

Lemma 1: [5] $w(t)$ and $q(t)$ are uniformly bounded above. And, the following lemma shows that $w(t)$ is ultimately bounded below.

Lemma 2: [5] There exists a $T_{L}>0$ such that $w(t) \geqslant \alpha$ for all $t>T_{L}$.

Note that the parameter $\alpha$ implies the expected number of backlogs on the link. That is, FAST TCP source sends at least $\alpha$ packets to the router buffer regardless of the network congestion status. The result of Lemma 2 agrees with this physical intuition.

\section{Stability ANALYSis}

In this section, we establish sufficient conditions for the global asymptotic stability of FAST TCP. As a preliminary, we present a following well-known stability result of the delaydifferential equation.

Lemma 3: Consider the delay-differential equation

$$
\dot{x}(t)=-a(t) x(t)-b(t) x(t-T(t)),
$$

where $a(t), b(t)$, and $T(t)$ are bounded continuous functions on $\mathbb{R}$. If $a(t) \geqslant \delta>0$, and there is a $k, 0 \leqslant k<1$ such that $|b(t)| \leqslant k \delta$, then the solution is uniformly asymptotically stable.

Proof: The proof is available from many references on delay-differential equations, e.g. [7, p. 154].

Using Lemma 3, we can easily derive a sufficient condition for the stability of FAST TCP.

Lemma 4: If $q(t)>d$, then the FAST TCP described by (3) is globally asymptotically stable.

Proof: Note that $\frac{q(t)}{d+q(t)}, \frac{d}{d+q(t)}$, and $T(t)$ are bounded continuous functions from Lemma 1 . Then, the proof is a direct application of Lemma 3 to (3).

However, since the condition of Lemma 4 is related not to the tuning parameters, but to the measured variable $q(t)$, it does not give much intuition on how to make the algorithm stable in a distributed way.
With the help of Lemma 2, Theorem 1 provides an improved condition that reveals the relationship between the parameter $\alpha$ and the stability of the algorithm.

Theorem 1: If $\alpha>c d$, then the FAST TCP (3) is globally asymptotically stable.

Proof: See Theorem 2 of [5].

Note that the derived condition $\alpha>c d$ is equivalent to $q^{*}=$ $\frac{\alpha}{c}>d$ which implies that not $q(t)$ for all $t$ but only the equilibrium queuing delay $q^{*}$ must be larger than $d$ to satisfy this condition.

From Lemma 4, if $q(t)>d$, then FAST TCP (3) is globally asymptotically stable. We now focus on the case of $q(t) \leqslant d$. From the Leibniz-Newton formula, the original model (3) can be transformed to

$$
\begin{aligned}
\dot{\tilde{w}}(t)= & -\gamma_{e} \tilde{w}(t)+\eta(t) \tilde{w}(t)-\eta(t) \tilde{w}(t-T) \\
= & -\gamma_{e} \tilde{w}(t)+\eta(t) \int_{-T}^{0} \dot{\tilde{w}}(t+\theta) d \theta \\
= & -\gamma_{e} \tilde{w}(t)-\eta(t) \int_{-T}^{0}\left(\gamma_{e}-\eta(t+\theta)\right) \tilde{w}(t+\theta) d \theta \\
& -\eta(t) \int_{-T}^{0} \eta(t+\theta) \tilde{w}(t-T+\theta) d \theta,
\end{aligned}
$$

where $\eta(t) \triangleq \gamma_{e} \frac{d}{d+q(t)}$. With the help of this model transformation technique, we derive another stability condition of FAST TCP in the following theorem. The condition reveals the relationship between the parameter $\gamma_{e}$ and the stability of the algorithm.

Theorem 2: If $\gamma_{e} d<\frac{2}{3}$, then the FAST TCP (3) is globally asymptotically stable.

Proof: If $q(t)>d$, then (3) is globally asymptotically stable from Lemma 4. Hence, in this proof, we focus on the case of $q(t) \leqslant d$ which makes $\eta(t)=\gamma_{e} \frac{d}{d+q(t)} \in\left[\frac{\gamma_{e}}{2}, \gamma_{e}\right]$ and $\gamma_{e}-\eta(t) \in\left[0, \frac{\gamma_{e}}{2}\right]$ for all $t$. If $V(\tilde{w}(t))=\tilde{w}^{2}(t) / 2$, then, whenever $|\tilde{w}(t)| \geqslant|\tilde{w}(t+\theta)|$ for all $\theta \in[-2 T, 0]$, we have

$$
\begin{aligned}
\dot{V}(\tilde{w}(t)) & \\
= & -\gamma_{e} \tilde{w}^{2}(t)-\eta(t) \tilde{w}(t) \int_{-T}^{0}\left(\gamma_{e}-\eta(t+\theta)\right) \tilde{w}(t+\theta) d \theta \\
& -\eta(t) \tilde{w}(t) \int_{-T}^{0} \eta(t+\theta) \tilde{w}(t-T+\theta) d \theta \\
\leqslant & -\gamma_{e} \tilde{w}^{2}(t)+\frac{\gamma_{e}}{2} \eta(t)|\tilde{w}(t)| \int_{-T}^{0}|\tilde{w}(t+\theta)| d \theta \\
& +\gamma_{e} \eta(t)|\tilde{w}(t)| \int_{-2 T}^{-T}|\tilde{w}(t+\theta)| d \theta \\
\leqslant & -\gamma_{e} \tilde{w}^{2}(t)+\frac{3}{2} \gamma_{e} \eta(t) \tilde{w}^{2}(t) T(t) \\
= & -\gamma_{e}\left(1-\frac{3}{2} \cdot \frac{\gamma_{e} d T(t)}{d+q(t)}\right) \tilde{w}^{2}(t) \\
= & -\gamma_{e}\left(1-\frac{3}{2} \gamma_{e} d\right) \tilde{w}^{2}(t),
\end{aligned}
$$

where the first inequality is from $\eta(t) \leqslant \gamma_{e}$ and $\gamma_{e}-\eta(t) \leqslant$ $\frac{1}{2} \gamma_{e}$ for all $t$. Therefore, if $\gamma_{e} d<\frac{2}{3}, \dot{V}(\tilde{w}(t)) \leqslant 0$ and we conclude from the Lyapunov-Razumikhin Theorem [7] that (3) is globally asymptotically stable.

From Theorem 1 and 2, we can conclude that a single-link single-source network with FAST TCP is globally asymptot- 


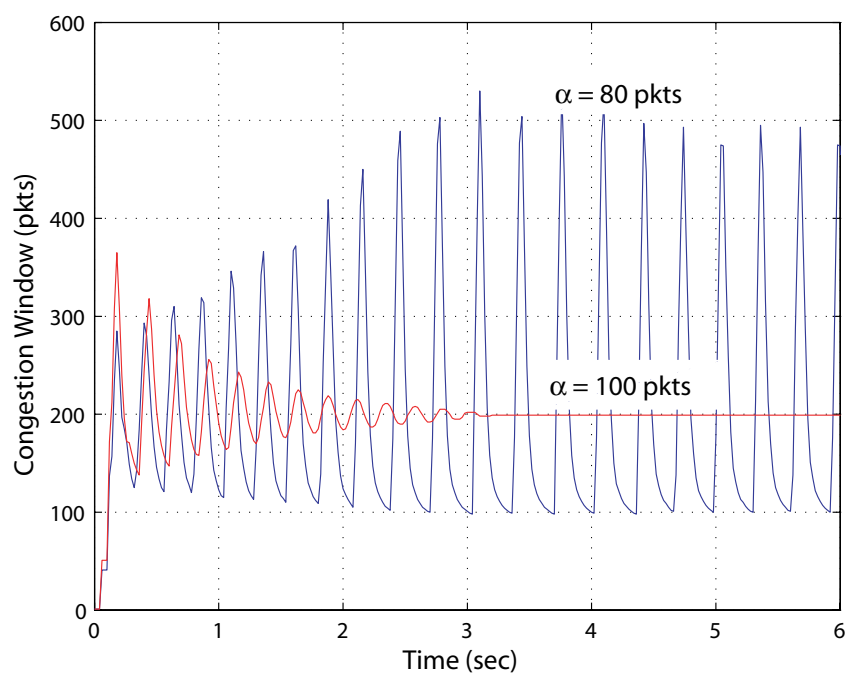

Fig. 1. Simulation validation of the condition $\alpha>c d$ ( $c=2 \mathrm{pkts} / \mathrm{ms}$, $d=50 \mathrm{~ms}, \gamma=0.5$, and $\Delta=5 \mathrm{~ms}$ ).

ically stable when, at least, one of the following conditions is satisfied: i) $\alpha>c d$, ii) $\gamma d<\frac{2}{3} \Delta$. These conditions can be satisfied in a decentralized way because each source can independently tune its own parameters $\alpha, \gamma$, and $\Delta$ based on its link status $c$ and $d$.

Remark 1: There are trade-offs between the stability conditions and the performance of the algorithm. In case of $\alpha>c d$, the condition requires sufficiently large $\alpha$ which induces large queuing delay, because the equilibrium queuing delay depends on $\alpha$ as $q^{*}=\frac{\alpha}{c}$. In case of $\gamma d<\frac{2}{3} \Delta$, the condition requires sufficiently small step size $\gamma$ or sufficiently large update period $\Delta$ which degrade the responsiveness of the algorithm.

Remark 2: In [3], [4], it is assumed that the FAST TCP source updates its congestion window once every RTT, i.e. $\Delta=d+q(t)$. In this case, it follows from Theorem 2 that FAST TCP is globally asymptotically stable with its default parameter setting $\gamma=0.5$, and this result agrees with the local stability results of [3], [4]. However, if each source updates its congestion window once every RTT, then the sources with the large RTT can suffer from unfairly slow update of their congestion window when they compete with the sources with the small RTT.

\section{Simulation}

We present a set of $n s-2$ [8] simulation results to illustrate the validity of the conditions for the global asymptotic stability of FAST TCP presented in Theorem 1 and 2. We adopted the code of [9] for the simulation of FAST TCP but slightly modified it to disable some features such as filter dynamics, pacing, and MI, which are not included in the model.

The first simulation test is conducted to validate the condition $\alpha>c d$. As shown in Fig. $1, \alpha$ is set to $c d$ for the (marginally) stable case, and a slightly smaller value than $c d$ for the unstable case. Fig. 1 illustrates that the congestion window asymptotically converges to its equilibrium point provided that $\alpha>c d$ and is oscillatory when $\alpha$ becomes slightly smaller than $c d$.

The second simulation test is conducted to validate the condition $\gamma d<\frac{2}{3} \Delta$. The parameter $\alpha$ is set to 10 to violate

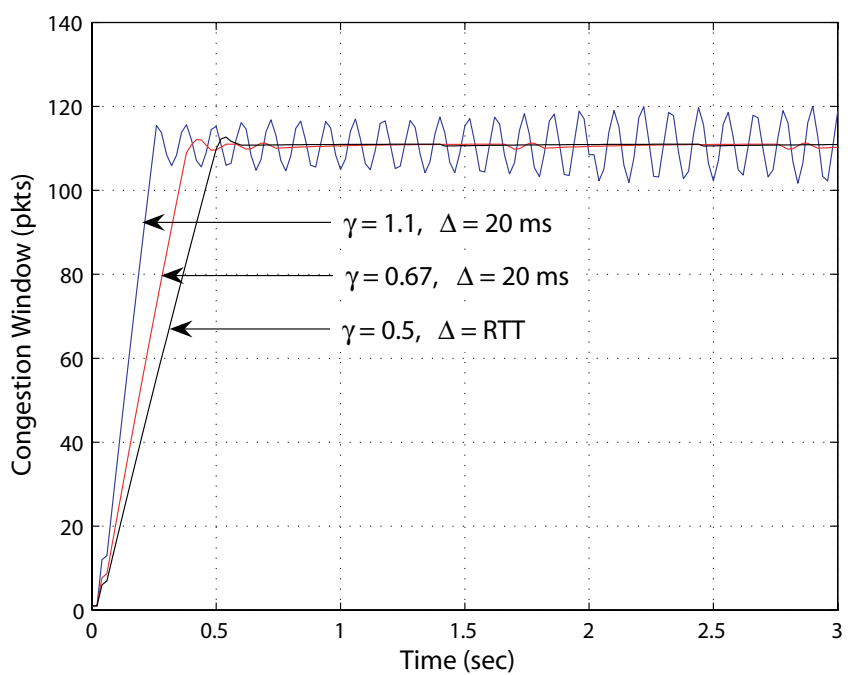

Fig. 2. Simulation validation of the condition $\gamma d<\frac{2}{3} \Delta(c=5 \mathrm{pkts} / \mathrm{ms}$, $d=20 \mathrm{~ms}$, and $\alpha=10 \mathrm{pkts}$ ).

the condition $\alpha>c d$ and the parameter $\gamma$ is set to satisfy $\gamma d<\frac{2}{3} \Delta$ for the stable case, and to a sufficiently large value for the unstable case. As shown in Fig. 2, if $\gamma$ is set to a sufficiently small value satisfying $\gamma d<\frac{2}{3} \Delta$, then the congestion window of the FAST TCP source asymptotically converges to its equilibrium point. Especially, if the congestion window update is performed once every RTT and $\gamma=0.5$, then FAST TCP is asymptotically stable.

\section{CONClusions}

In this letter, we have derived sufficient conditions for the global asymptotic stability of FAST TCP in a singlelink single-source network with network feedback delays. The derived conditions are explicitly appeared in terms of all the tuning parameters of FAST TCP such as $\alpha, \gamma$, and the window update period. The conditions are verified by simulation experiments. These conditions can be satisfied in a decentralized way because each source can independently tune its own parameters based on its link status.

\section{REFERENCES}

[1] D. X. Wei, C. Jin, S. H. Low, and S. Hegde, "FAST TCP: motivation, architecture, algorithms, performance," IEEE/ACM Trans. Networking, vol. 14, pp. 1246-1259, Dec. 2006.

[2] J. Wang, D. X. Wei, and S. H. Low, "Modelling and stability of FAST TCP," in Proc. IEEE Infocom 2005, Apr. 2005.

[3] A. Tang, K. Jacobsson, L. L. H. Andrew, and S. H. Low, "An accurate link model and its application to stability analysis of FAST TCP," in Proc. IEEE Infocom 2007, May 2007.

[4] L. Tan, W. Zhang, and C. Yuan, "On parameter tuning for FAST TCP," IEEE Commun. Lett., vol. 11, no. 5, pp. 458-460, May 2007.

[5] J.-Y. Choi, K. Koo, J. S. Lee, and S. H. Low, "Global stability of FAST TCP in single-link single-source network," in Proc. 44th IEEE Conf. Decision Control, Dec. 2005.

[6] K. Koo, J.-Y. Choi, and J. S. Lee, "Two different models of FAST TCP and their stable and efficient modification," in Proc. NET-COOP 2007, LNCS 4465, pp. 65-73, June 2007.

[7] J. K. Hale and S. M. V. Lunel, Introduction to Functional Differential Equations, 3rd ed. Prentice Hall, 1993.

[8] The network simulator. [Online]. http://www.isi.edu/nsnam/ns.

[9] FAST TCP simulator module for $n s-2$, version 1.1. [Online]. Available: http://www.cubinlab.ee.mu.oz.au/ns2fasttcp. 